\title{
ELEMENTARY METHODS FOR FAILURE DUE TO A SEQUENCE OF MARKOVIAN EVENTS
}

\author{
J. GANI \\ Australian National University \\ School of Mathematical Sciences \\ Canberra ACT 0200, Australia
}

(Received October, 1997; Revised January, 1998)

\begin{abstract}
This paper is concerned with elementary methods for evaluating the distribution of the time to system failure, following a particular sequence of events from a Markov chain. After discussing a simple example in which a specific sequence from a two-state Markov chain leads to failure, the method is generalized to a sequence from a $(k>2)$-state chain. The expectation and variance of the time $T$ to failure can be obtained from the probability generating function (p.g.f.) of $T$. The method can be extended to the case of continuous time.
\end{abstract}

Key words: Failure Time, Markov Chain, Augmented Transition Probability Matrix, Sequence Overlap, Continuous Time.

AMS subject classifications: $60 \mathrm{~J} 10,60 \mathrm{~J} 27,90 \mathrm{~B} 25$.

\section{Introduction}

In many processes arising in reliability theory, a system may at any time $t=$ $0,1,2, \ldots$, be in one of $k \geq 2$ states $A_{1}, A_{2}, \ldots, A_{k}$, the sequence of which forms a Markov chain. While this chain can be simple or of order $r \geq 2$, we restrict ourselves here to simple Markov chains; any chain of higher order can be reduced to a simple chain by redefining the states.

A system usually fails after it has passed through a particular sequence $A_{i_{1}}, A_{i_{2}}, \ldots, A_{i_{m}}$ of $m$ states $(m \geq k$, or $<k$ ) at the times $T-m+1, T-m+2$, $\ldots, T$, with possible repetitions of states, before failure at $T \geq m$. We wish to study the distribution of the failure time $T$.

In order to formulate the problem clearly and solve it, we rely on the approaches of Blom and Thorburn [1], Fu and Koutras [3], and Gani [4]. In a sense, the methods used are an extension of the theory of runs first studied by Mood [6] and Feller [2, Chapter 2]. These methods have been considered by Guibas and Odlyzko [5], and are applied here to Markov chains rather than independent trials. While our results cannot claim great originality, they have the virtue of being elementary, thus making them readily accessible to engineers and operations researchers. 


\section{The Case of the 2-State Chain}

When $k=2$, we may for simplicity label the states $A_{1}, A_{2}$ as 0,1 , forming the simple Markov chain with transition probability matrix

$$
\begin{gathered}
X_{n+1}=0 \\
0 \\
X_{n}=\left[\begin{array}{cc}
p_{00} & p_{01} \\
p_{10} & p_{11}
\end{array}\right],
\end{gathered}
$$

and initial probabilities $\left[p_{0}, p_{1}\right]$ where $P\left\{X_{0}=0\right\}=p_{0}, P\left\{X_{0}=1\right\}=p_{1}$.

Let us assume that failure occurs when the sequence 010 arises for the first time. We now form an augmented transition probability matrix for the states $0,1,01,010$, this last being an absorbing state:

$$
\begin{aligned}
& X_{n+1}=\begin{array}{llll}
0 & 1 & 01 & 010
\end{array} \\
& X_{n}=\begin{array}{c}
1 \\
01 \\
010
\end{array}\left[\begin{array}{ccc|c}
p_{00} & 0 & p_{01} & 0 \\
p_{10} & p_{11} & 0 & 0 \\
0 & p_{11} & 0 & p_{10} \\
\cline { 2 - 5 } & 0 & 0 & 1
\end{array}\right]=\left[\begin{array}{c|c}
P & Q \\
0 & 1
\end{array}\right] \text {. }
\end{aligned}
$$

If we denote the initial probability vector by $p^{\prime}=\left[p_{0} p_{1} 0\right]$, we can readily see that the failure time $T_{010}$ will have the probability distribution

$$
P\left\{T_{010}=n\right\}=p^{\prime} P^{n-2} Q, \quad n \geq 2,
$$

where in fact this probability is 0 for $n<3$.

The probability generating function (p.g.f.) of $T_{010}$ is

$$
f_{010}(\theta)=\sum_{n=2}^{\infty} p^{\prime} \theta(P \theta)^{n-2} Q \theta, \quad 0<\theta \leq 1 .
$$

This can be derived explicitly as

$$
\begin{aligned}
f_{010}(\theta)= & p^{\prime} \theta[I-P \theta]^{-1} Q \theta=\left[\begin{array}{lcc}
p_{0} \theta & p_{1} \theta & 0
\end{array}\right] \frac{1}{|I-P \theta|} \\
\times & {\left[\begin{array}{ccc}
1-p_{11} \theta & p_{01} p_{11} \theta^{2} & p_{01} \theta\left(1-p_{11} \theta\right) \\
p_{10} \theta & 1-p_{00} \theta & p_{01} p_{10} \theta^{2} \\
p_{10} p_{11} \theta^{2} & \left(1-p_{00} \theta\right) p_{11} \theta & \left(1-p_{00} \theta\right)\left(1-p_{11} \theta\right)
\end{array}\right]\left[\begin{array}{c}
0 \\
0 \\
p_{10} \theta
\end{array}\right] } \\
& =\frac{\theta^{3}\left[p_{0} p_{01} p_{10}+\theta\left(p_{1} p_{01} p_{10}^{2}-p_{0} p_{01} p_{10} p_{11}\right)\right]}{1-\left(p_{00}+p_{11}\right) \theta+p_{00} p_{11} \theta^{2}-p_{01} p_{10} p_{11} \theta^{3}}
\end{aligned}
$$


One can easily find the expectation and variance of $T_{010}$ from (2.5) as

$$
E\left(T_{010}\right)=f_{010}^{\prime}(1), V\left(T_{010}\right)=f_{010}^{\prime \prime}(1)+f_{010}^{\prime}(1)-\left(f_{010}^{\prime}(1)\right)^{2}
$$

Such calculations can be tedious if the full notation is retained, but become simpler if specific values are used for the probabilities, as in the following example.

Example 2.1: Let $p_{0}=p_{1}=0.5, p_{00}=0.7$ and $p_{01}=0.3, p_{10}=0.4$ and $p_{11}=$ 0.6 ; we then find from $(2.5)$ that

$$
\begin{aligned}
& f_{010}(\theta)=\frac{\theta^{3}[0.06-0.12 \theta]}{1-1.3 \theta+0.42 \theta^{2}-0.072 \theta^{3}} \\
& f_{010}^{\prime}(\theta)=\frac{\theta^{2}\left[0.18-0.204 \theta+0.072 \theta^{2}-0.01008 \theta^{3}+0.000864 \theta^{4}\right]}{\left[1-1.3 \theta+0.42 \theta^{2}-0.072 \theta^{3}\right]^{2}}, \\
& f_{010}^{\prime \prime}(\theta)=\frac{\theta\left[0.36-0.612 \theta+0.402 \theta^{2}-0.10008 \theta^{3}+0.000432 \theta^{4}-0.0018592 \theta^{5}\right]}{\left[1-1.3 \theta+0.42 \theta^{2}-0.072 \theta^{3}\right]^{3}} .
\end{aligned}
$$

It follows that

$$
\begin{gathered}
E\left(T_{010}\right)=f_{010}^{\prime}(1)=16.8333 \\
V\left(T_{010}\right)=f_{010}^{\prime \prime}(1)+f_{010}^{\prime}(1)-\left(f_{010}^{\prime}(1)\right)^{2}=205.5788
\end{gathered}
$$

so that $\sigma\left(T_{010}\right)=14.3380$.

This indicates how large the variation can be in the time $T_{010}$ until failure occurs; the range of $E\left(T_{010}\right) \pm \sigma\left(T_{010}\right)$ is 2.4953 to 31.1713 , with $T_{010} \geq 3$. It should be pointed out that if the sequence required for failure had been 000 , the augmented transition probability matrix would have been

$$
\begin{aligned}
& X_{n+1}=\begin{array}{llll}
0 & 1 & 00 & 000
\end{array} \\
& X_{n}=\begin{array}{c}
0 \\
1 \\
00 \\
000
\end{array}\left[\begin{array}{ccc|c}
0 & p_{01} & p_{00} & 0 \\
p_{10} & p_{11} & 0 & 0 \\
0 & p_{01} & 0 & p_{00} \\
& 0 & 0 & 1
\end{array}\right]=\left[\begin{array}{c|c}
P & Q \\
0 & 1
\end{array}\right] \text {. }
\end{aligned}
$$

with the same transition probabilities as in (2.1), and the same initial probabilities $p_{0}$ and $p_{1}$. The submatrices $P, Q$ of $(2.9)$ are now different from those in (2.2), with the result that the p.g.f. $f_{000}(\theta)$ of $T_{000}$ differs from $f_{010}(\theta)$ of $(2.5)$, as do also the relevant expectation and standard deviation.

\section{An $m$-Sequence of States for $k>2$}

Let us now suppose that the sequence of states leading to failure is $A_{i_{1}} A_{i_{2}} \ldots A_{i_{m}}$ where these $m$ states $(m \geq k$, or $<k)$ are selected from among the $k>2$ states $A_{1}$, 
$A_{2}, \ldots, A_{k}$. These form a Markov chain with transition probability matrix

$$
\begin{aligned}
& X_{n+1}=\begin{array}{llll}
A_{1} & A_{2} & \ldots & A_{k}
\end{array} \\
& X_{n}=\begin{array}{c}
A_{1} \\
A_{2} \\
\vdots \\
A_{k}
\end{array}\left[\begin{array}{ccc}
p_{11} & p_{12} & p_{1 k} \\
p_{21} & p_{22} & p_{2 k} \\
\vdots & \vdots & \vdots \\
p_{k 1} & p_{k 2} & p_{k k}
\end{array}\right] \text {. }
\end{aligned}
$$

and initial probabilities $p_{i}=P\left\{X_{0}=i\right\}, i=1, \ldots, k$. We consider the augmented transition probability matrix for the states $A_{1}, A_{2}, \ldots, A_{k}, A_{i_{1}} A_{i_{2}}, A_{i_{1}} A_{i_{2}} A_{i_{3}}, \ldots$, $A_{i_{1}} A_{i_{2}} \ldots A_{i_{m}}$, this last being an absorbing state:

$$
X_{n+1}=A_{1} \ldots A_{i_{1}} \ldots A_{i_{2}} \ldots A_{i_{3}} \ldots A_{k} A_{i_{1}} A_{i_{2}} A_{i_{1}} A_{i_{2}} A_{i_{3}} \ldots A_{i_{1}} \ldots A_{i_{m}}
$$

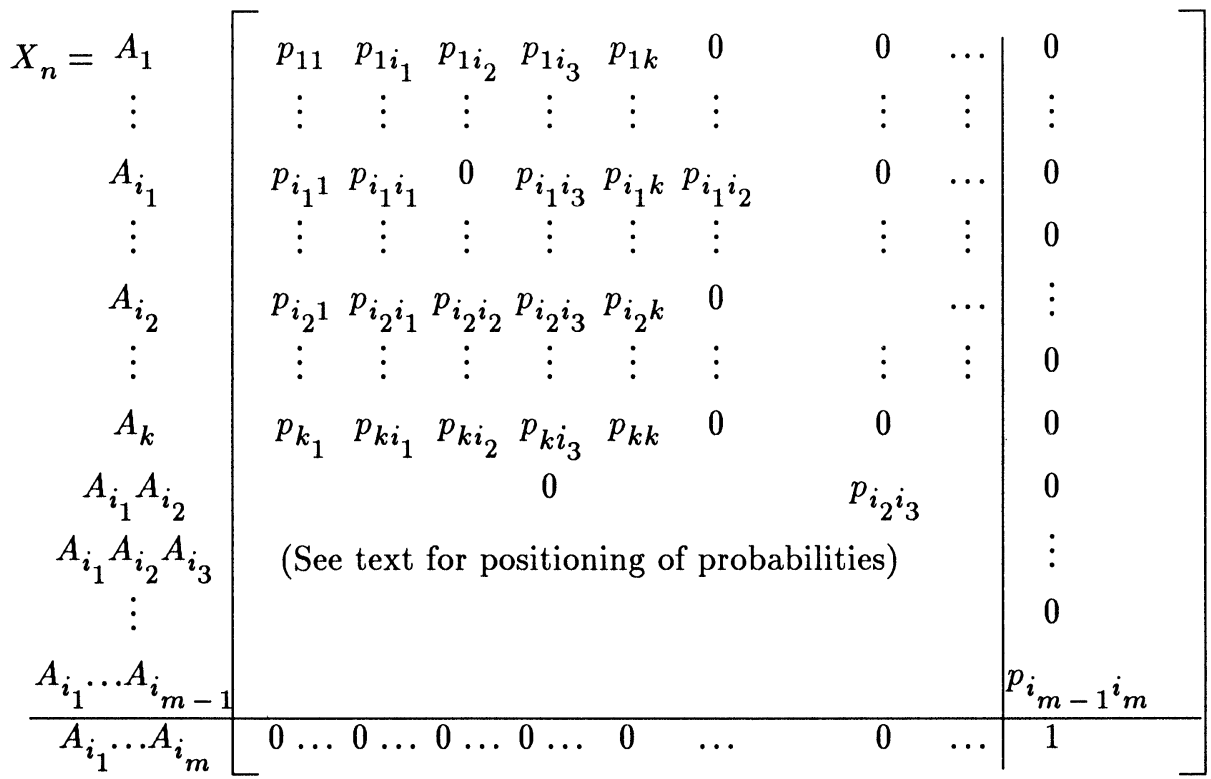

$$
\begin{aligned}
& =\left[\begin{array}{l|l}
P & Q \\
0 & 1
\end{array}\right] \text {. }
\end{aligned}
$$

We note that in the submatrix for the states $A_{1}, \ldots, A_{i_{1}}, \ldots, A_{i_{2}}, \ldots, A_{i_{3}}, \ldots, A_{k}$, all probabilities except $p_{i_{1} i_{2}}$ are positioned as in (3.1), but $P\left\{A_{i_{1}} \rightarrow A_{i_{1}} A_{i_{2}}\right\}$ is $p_{i_{1} i_{2}}$ positioned at $\left(i_{1}, i_{1} i_{2}\right)$ so that $P\left\{A_{i_{1}} \rightarrow A_{i_{2}}\right\}=0$ at $\left(i_{1}, i_{2}\right)$. Next, $P\left\{A_{i_{1}} A_{i_{2}} \rightarrow\right.$ $\left.A_{i_{1}} A_{i_{2}} A_{i_{3}}\right\}=p_{i_{2} i_{3}}$ is positioned at $\left(i_{1} i_{2}, i_{1} i_{2} i_{3}\right)$ so that $P\left\{A_{i_{1}} A_{i_{2}} \rightarrow A_{i_{3}}\right\}=0$ at $\left(i_{1} i_{2}, i_{3}\right)$. Also, if $A_{i_{1}} A_{i_{2}}$ is $A_{i_{1}} A_{i_{1}}$ and the next state is also $A_{i_{1}}$, then $A_{i_{1}} A_{i_{2}} A_{i_{1}}=$ $A_{i_{1}} A_{i_{1}} A_{i_{1}}$ with the last 2 states overlapping the first two. In this case

$$
P\left\{A_{i_{1}} A_{i_{1}} \rightarrow A_{i_{1}} A_{i_{1}}\right\}=p_{i_{1} i_{1}}
$$


is positioned at $\left(i_{1} i_{1}, i_{1} i_{1}\right)$ with $P\left\{A_{i_{1}} A_{i_{1}} \rightarrow A_{i_{1}}\right\}=0$.

We outline a general method for allocating the transition probabilities in the lower part of the matrix (3.2) for the states $A_{i_{1}} A_{i_{2}}, \ldots, A_{i_{1}} \ldots A_{i_{m-1}}$. Suppose we are in the state $X_{n}=A_{i_{1}} \ldots A_{i_{j}}$, then $X_{n+1}$ will be one of $A_{i_{1}} \ldots A_{i_{j}} A_{1}, A_{i_{1}} \ldots A_{i_{j}} A_{2}, \ldots$ $A_{i_{1}} \ldots A_{i_{j}} A_{i_{j+1}}, \ldots, A_{i_{1}} \ldots A_{i_{j}} A_{k}$. Clearly

$$
P\left\{A_{i_{1}} \ldots A_{i_{j}} \rightarrow A_{i_{1}} \ldots A_{i_{j}} A_{i_{j+1}}\right\}=p_{i_{j} i_{j+1}}
$$

will be positioned at $\left(i_{1} \ldots i_{j}, i_{1} \ldots i_{j} i_{j+1}\right)$ with $P\left\{A_{i_{1}} \ldots A_{i_{j}} \rightarrow A_{i_{j+1}}\right\}=0$ at $\left(i_{1} \ldots i_{j}, i_{j+1}\right)$. For all other states of $X_{n+1}$ not ending with $A_{i_{j+1}}$, we have in the position $\left(i_{1} i_{2} \ldots i_{j}, l\right)$ the probability

$$
P\left\{A_{i_{1}} \ldots A_{i_{j}} \rightarrow A_{l}\right\}=p_{i_{j} l} \quad\left(l \neq i_{j+1}\right)
$$

with the following exceptions. For some values of $r(2 \leq r \leq j)$ there may be an overlap between the first and last $j+2-r$ states of $A_{i_{1}} A_{i_{2}} \ldots A_{i_{r}} \ldots A_{i_{j}} A_{l}$, so that

$$
A_{i_{1}} A_{i_{2}} \ldots A_{i_{j+2-r}}=A_{i_{r}} \ldots A_{i_{j}} A_{l} \text {. }
$$

In this case, the probability $p_{i_{j} l}$ is allocated to the position $\left(i_{1} \ldots i_{j}, i_{1} \ldots i_{j+2-r}\right)$ with the consequence that $P\left\{A_{i_{1}} \ldots A_{i_{j}} \rightarrow A_{l}\right\}=0$.

Once the matrix (3.2) is fully defined, we have that the time to failure $T_{i_{1} i_{2} \ldots{ }_{m}}$ follows the distribution

$$
P\left\{T_{i_{1} i_{2} \ldots i_{m}}=n\right\}=p^{\prime} P^{n-2} Q, \quad n \geq 2 .
$$

Here $p^{\prime}=\left[\begin{array}{lllll}p_{1} & p_{2} & \ldots & p_{k} & 0 \ldots 0\end{array}\right]$ is the $1 \times(k+m-2)$ vector of initial probabilities, $P$ is the $(k+m-2) \times(k+m-2)$ matrix in $(3.2)$ and $Q$ the $(k+m-2) \times 1$ column vector with zero components, except for the last row whose entry is $p_{i_{m-1}{ }^{i} m}$. Note that $T_{i_{1} \ldots i_{m}} \geq m$.

The p.g.f. $f_{i_{1} \ldots i_{m}}(\theta)$ of $T_{i_{1} \ldots i_{m}}$ is

$$
f_{i_{1} \ldots i_{m}}(\theta)=p^{\prime} \theta[I-P \theta]^{-1} Q \theta
$$

which can, in principle, be evaluated if the structure of $P$ is known. But it is clearly not possible to give an explicit result which will hold generally.

Perhaps the procedure is best illustrated by an example for a Markov chain with $k=3$ states $A_{1}, A_{2}, A_{3}$, and a sequence of $m=3$ states, say $A_{2} A_{2} A_{3}$, leading to failure. The basic transition probability matrix is

$$
\begin{gathered}
X_{n+1}=\begin{array}{lll}
A_{1} & A_{2} & A_{3} \\
A_{1} \\
X_{n}= \\
A_{2} \\
A_{3}
\end{array}\left[\begin{array}{lll}
p_{11} & p_{12} & p_{13} \\
p_{21} & p_{22} & p_{23} \\
p_{31} & p_{32} & p_{33}
\end{array}\right],
\end{gathered}
$$


with initial probabilities $P\left\{X_{0}=A_{i}\right\}=p_{i}, i=1,2,3$. The augmented matrix for the states $A_{1}, A_{2}, A_{3}, A_{2} A_{2}, A_{2} A_{2} A_{3}$ is given by

$$
\begin{aligned}
& X_{n+1}=A_{1} \quad A_{2} \quad A_{3} \quad A_{2} A_{2} \quad A_{2} A_{2} A_{3} \\
& X_{n}=\begin{array}{c}
A_{1} \\
A_{2} \\
A_{3} \\
A_{2} A_{2} \\
A_{2} A_{2} A_{3}
\end{array}\left[\begin{array}{cccc|c}
p_{11} & p_{12} & p_{13} & 0 & 0 \\
p_{21} & 0 & p_{23} & p_{22} & 0 \\
p_{31} & p_{32} & p_{33} & 0 & 0 \\
p_{21} & 0 & 0 & p_{22} & p_{23} \\
\hline 0 & 0 & 0 & 0 & 1
\end{array}\right]=\left[\begin{array}{c|c}
P & Q \\
0 & 1
\end{array}\right] .
\end{aligned}
$$

Note that $P\left\{A_{2} \rightarrow A_{2} A_{2}\right\}=p_{22}$ with the consequence that $P\left\{A_{2} \rightarrow A_{2}\right\}=0$; also, in going from $X_{n}=A_{2} A_{2}$ to $X_{n+1}=A_{2} A_{2} A_{2}$, since the last $A_{2} A_{2}$ of $X_{n+1}$ overlap the $A_{2} A_{2}$ of $X_{n}$, we have $P\left\{A_{2} A_{2} \rightarrow A_{2} A_{2}\right\}=p_{22}$ with $P\left\{A_{2} A_{2} \rightarrow A_{2}\right\}=0$.

Exactly as in (3.7), the distribution of the failure time $T_{223}$ is

$$
P\left\{T_{223}=n\right\}=\left[\begin{array}{lll}
p_{1} & p_{2} & p_{3}
\end{array}\right] P^{n-2} Q, n \geq 2,
$$

where this probability is 0 for $n<3$. The p.g.f. of $T_{223}$ is

$$
\begin{aligned}
f_{223}(\theta) & =\sum_{n=2}^{\infty}\left[\begin{array}{llll}
p_{1} \theta & p_{2} \theta & p_{3} \theta & 0
\end{array}\right](P \theta)^{n-2} Q \theta \\
& =p^{\prime} \theta[I-P \theta]^{-1} Q \theta, \quad 0<\theta \leq 1 .
\end{aligned}
$$

In its explicit form, this is

$$
f_{223}(\theta)=\left[\begin{array}{lll}
p_{1} \theta & p_{2} \theta & p_{3} \theta
\end{array}\right] \frac{1}{|I-P \theta|}\left[\begin{array}{cccc}
\cdot & \cdot & \cdot & A_{14} \\
\cdot & \cdot & \cdot & A_{24} \\
\cdot & \cdot & \cdot & A_{34} \\
\cdot & \cdot & \cdot & \cdot
\end{array}\right]\left[\begin{array}{c}
0 \\
0 \\
0 \\
p_{23} \theta
\end{array}\right]
$$

where the dots indicate that the values of these elements are irrelevant to our calculations. We find that

$$
\begin{gathered}
A_{14}=\theta^{2}\left[p_{13} p_{22} p_{32} \theta+p_{12} p_{22}\left(1-p_{33} \theta\right)\right] \\
A_{24}=\theta\left[p_{22}\left(1-p_{11} \theta\right)\left(1-p_{33} \theta\right)-p_{13} p_{22} p_{31} \theta^{2}\right] \\
A_{34}=\theta^{2}\left[p_{12} p_{22} p_{31} \theta+p_{22} p_{32}\left(1-p_{11} \theta\right)\right] \\
|I-P \theta|=\left(1-p_{22} \theta\right)\left[\left(1-p_{11} \theta\right)\left(1-p_{33} \theta\right)\right. \\
-\theta^{2}\left\{p_{13} p_{31}+p_{12} p_{21}\left(1-p_{33} \theta\right)+p_{23} p_{32}\left(1-p_{11} \theta\right)\right\} \\
\left.-\theta^{3}\left(p_{12} p_{23} p_{31}+p_{13} p_{21} p_{32}\right)\right]-p_{22} p_{21} \theta^{3}\left[p_{13} p_{32} \theta+p_{12}\left(1-p_{33} \theta\right)\right] .
\end{gathered}
$$


This finally yields

$$
f_{223}(\theta)=\frac{p_{23} p_{1} \theta^{2} A_{14}+p_{23} p_{2} \theta^{2} A_{24}+p_{23} p_{3} \theta^{2} A_{34}}{|I-P \theta|}
$$

where the numerator is $\theta^{3}$ times a quadratic polynomial, and $|I-P \theta|$ is a quartic polynomial. Once again, we use specific values of the probabilities to illustrate our procedure by an example.

Example 3.1: Let $p_{1}=p_{2}=p_{3}=0.3333$, and the matrix (3.7) be

It follows from (3.13) that

$$
\left[\begin{array}{lll}
0.4 & 0.3 & 0.3 \\
0.6 & 0.3 & 0.1 \\
0.5 & 0.2 & 0.3
\end{array}\right] .
$$

$$
f_{223}(\theta)=\frac{0.00999 \theta^{3}\left[1-0.2 \theta+0.01 \theta^{2}\right]}{1-\theta-0.02 \theta^{2}+0.026 \theta^{3}+0.0021 \theta^{4}} .
$$

By differentiating $f_{223}(\theta)$ and its first derivative, we obtain

$$
\begin{gathered}
E\left(T_{223}\right)=f_{223}^{\prime}(1)=120.3857 \\
V\left(T_{223}\right)=f_{223}^{\prime \prime}(1)+f_{223}^{\prime}(1)-\left(f_{223}^{\prime}(1)\right)^{2}=13960.5636
\end{gathered}
$$

so that $\sigma\left(T_{223}\right)=118.1548$.

Once again, as in Example 2.1, the variation in $T_{223}$ is very large; the range of $E\left(T_{223}\right) \pm \sigma\left(T_{223}\right)$ is 2.2309 to 238.5405 , with $T_{223} \geq 3$.

\section{The Continuous Time Case}

In most reliability problems, events occur in continuous time $t \geq 0$, with changes of state arising at points arriving in a Poisson process with parameter $\lambda$. The discrete time process of Section 3 may be regarded as one embedded in the Poisson process. In this case, the probability that the failure time $T_{i_{1} i_{2} \ldots i_{m}}$ lies in $(t, t+\delta t)$ after states $A_{i_{1}} A_{i_{2}} \ldots A_{i_{m}}$ have occurred in this sequence for the first time, will be

$$
g(t) \delta t=P\left\{t \leq T_{i_{1} \ldots i_{m}}<t+\delta t\right\}=\sum_{n=2}^{\infty} e^{-\lambda t} \frac{(\lambda t)^{n-1}}{(n-1) !} \lambda\left[p^{\prime} P^{n-2} Q\right] \delta t,
$$

where $p^{\prime}=\left[\begin{array}{lllll}p_{1} & p_{2} \ldots p_{k} & 0 & 0 . .0\end{array}\right]$ is a $1 \times(k+m-2)$ vector of initial probabilities, $P$ is the $(k+m-2) \times(k+m-2)$ matrix in $(3.2)$ and $Q$ the $(k+m-2) \times 1$ column vector whose single non-zero entry is $p_{i_{m-1}{ }^{i} m}$ in the last row.

It is simpler to deal with the Laplace transform in (4.1); this is given by

$$
\widehat{g}(s)=\int^{\infty} e^{-s t} \sum_{n=2}^{\infty} e^{-\lambda t} \frac{(\lambda t)^{n-1}}{(n-1) !} \lambda\left[p^{\prime} P^{n-2} Q\right] d t, \text { Re } s \geq 0
$$




$$
=\sum_{n=2}^{\infty}\left(\frac{\lambda}{\lambda+s}\right)^{n} p^{\prime} P^{n-2} Q=\left(\frac{\lambda}{\lambda+s}\right)^{2} p^{\prime}\left(I-\frac{\lambda}{\lambda+s} P\right)^{-1} Q .
$$

We proceed to illustrate its use by an example.

Example 4.1: We refer to the Example 2.1, which we now reconsider in continuous time, the changes of state occurring at the points of a Poisson process with parameter $\lambda$. The Laplace transform of the failure time $T_{010}$ in this case is

$$
\widehat{g}(s)=\left(\frac{\lambda}{\lambda+s}\right)^{3} \frac{0.06-0.012 \frac{\lambda}{\lambda+s}}{1-1.3 \frac{\lambda}{\lambda+s}+0.42\left(\frac{\lambda}{\lambda+s}\right)^{2}-0.072\left(\frac{\lambda}{\lambda+s}\right)^{3}} .
$$

Thus, the distribution of $T_{010}$ is an infinite sum of gamma type variables starting with those having Laplace transforms

$$
0.06\left(\frac{\lambda}{\lambda+s}\right)^{3}-0.012\left(\frac{\lambda}{\lambda+s}\right)^{4}
$$

or probability density functions

$$
0.06 \lambda \frac{(\lambda t)^{2}}{2 !} e^{-\lambda t}-0.012 \lambda \frac{(\lambda t)^{3}}{3 !} e^{-\lambda t}
$$

The next set of terms will be

$$
0.078 \lambda \frac{(\lambda t)^{3}}{3 !} e^{-\lambda t}-0.0156 \lambda \frac{(\lambda t)^{4}}{4 !} e^{-\lambda t}
$$

as we begin to expand the denominator of (4.3). While the method is simple to describe, it is rather intractable when $k$ and $m$ are large.

\section{References}

[1] Blom, G. and Thorburn, D., How many random digits are required until given sequences are obtained, J. Appl. Prob. 19 (1982), 518-531.

[2] Feller, W., An Introduction to Probability Theory and its Applications, 3rd Ed., Wiley, New York 1968.

[3] Fu, J.C. and Koutras, M.V., Distribution theory of runs: a Markov chain approach, J. Amer. Statist. Assoc. 89 (1994), 1050-1058.

[4] Gani, J., On sequences of events with repetitions, Stoch. Models 14 (1998), to appear.

[5] Guibas, L.J. and Odlyzko, A.M., String overlaps, pattern matching and nontransitive games, J. Combin. Theory A30 (1981), 181-208.

[6] Mood, A.M., The distribution theory of runs, Ann. Math. Statist. 11 (1940), 367-392. 


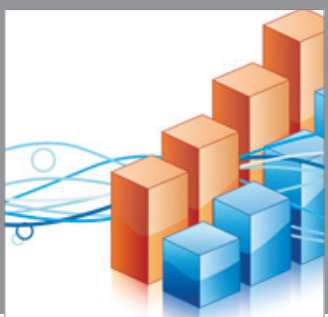

Advances in

Operations Research

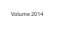

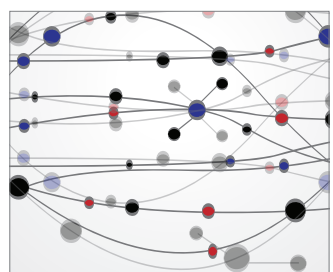

\section{The Scientific} World Journal
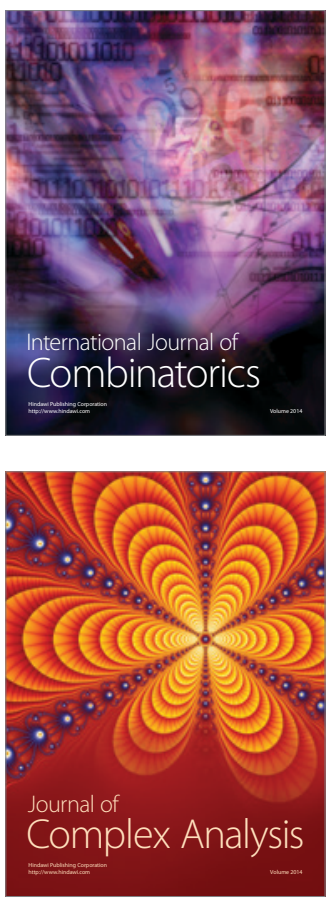

International Journal of

Mathematics and

Mathematical

Sciences
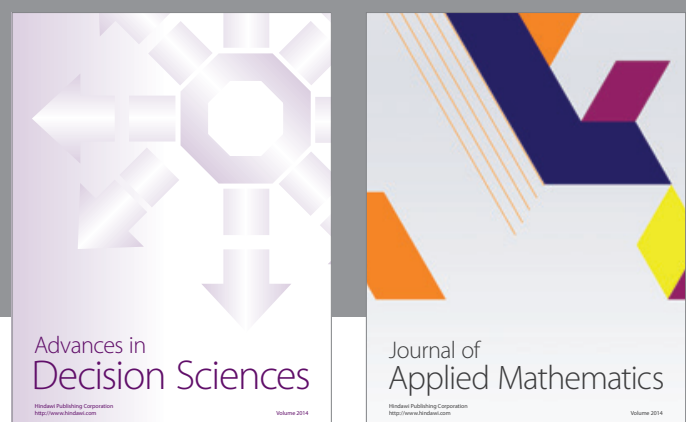

Journal of

Applied Mathematics
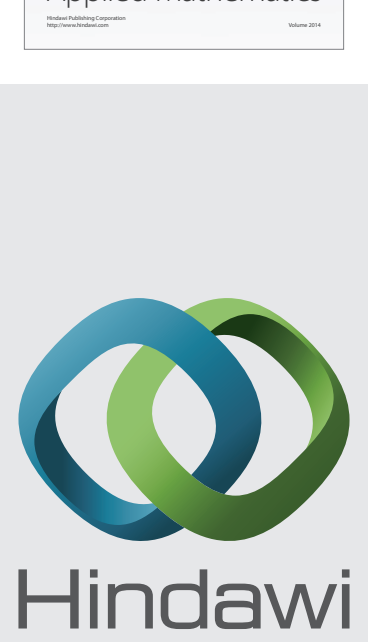

Submit your manuscripts at http://www.hindawi.com
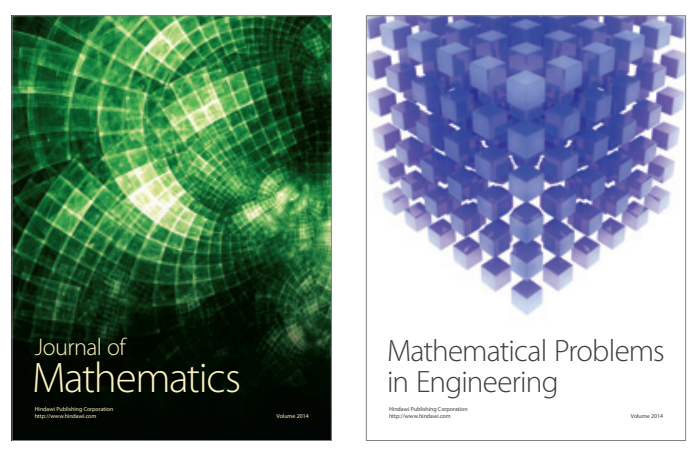

Mathematical Problems in Engineering
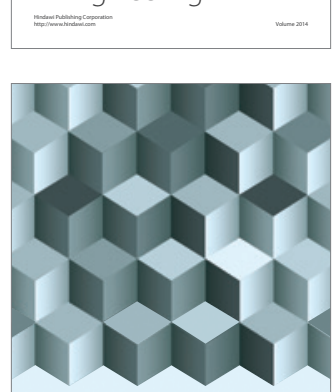

Journal of

Function Spaces
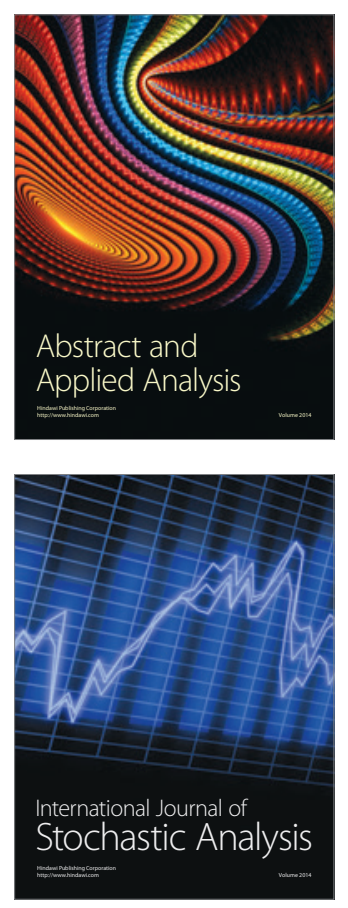

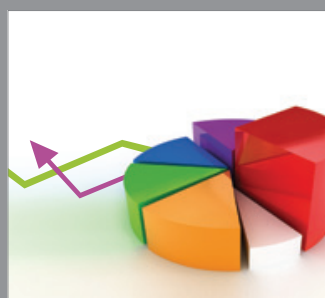

ournal of

Probability and Statistics

Promensencen
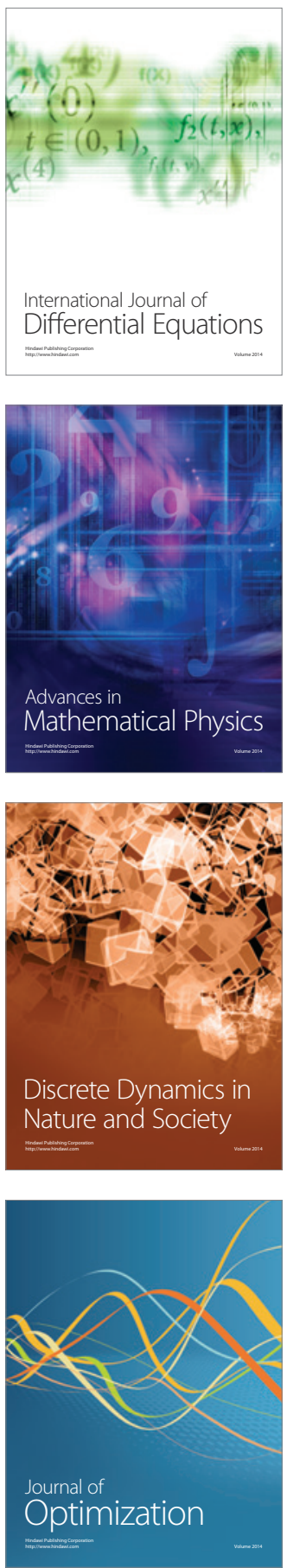